\title{
Optimal Replenishment and Lot Sizing Strategy for Inventory Mechanism with Step-Shaped Demand and Backordering
}

\author{
Lixia Zhang $\mathbb{D}^{1}$ and Lili Han $\mathbb{D}^{2}$ \\ ${ }^{1}$ Department of Mathematics, Jining University, Qufu, Shandong, 273155, China \\ ${ }^{2}$ School of Management Science, Qufu Normal University, Rizhao, Shandong, 276800, China \\ Correspondence should be addressed to Lili Han; hllrz@163.com
}

Received 19 April 2019; Accepted 9 July 2019; Published 4 August 2019

Academic Editor: Chaudry M. Khalique

Copyright (c) 2019 Lixia Zhang and Lili Han. This is an open access article distributed under the Creative Commons Attribution License, which permits unrestricted use, distribution, and reproduction in any medium, provided the original work is properly cited.

This paper discusses the inventory mechanism with backordering and with the infinite planning horizon consisting of two stages wherein the demand rate in the first stage is strictly greater than that in the second stage. To minimize the retailer's inventory cost, we establish a lot sizing decision model. On the basis of the inventory cost analysis, we present a closed-form solution to the model and provide an optimal replenishment and stocking strategy to the retailer. The given numerical experiments show the validity of the model.

\section{Introduction}

The economic order quantity (EOQ) is a seminal inventory model which was originally introduced by Harris [1] and further developed by Wilson [2]. Almost 50 years later, many variations were developed that differ in assumptions on not only the life time of an item, but also the type of demand, the presence of price discounts, allowing shortages and backordering, single or multiple items, one or two warehouses, single or multiechelon modeling, average cost or discounted cash flows, and whether a delay in payment is permissible in Bakker [3]. An elaborate review of the model and its well-known extensions can be found in Nahmias [4]. To make the model more realistic, various relaxations on demand rate are made thereafter by Waters [5]. The extensions of the basic EOQ model include inventory models with a limited-time price incentives such as a one-time-only or temporary price reduction and a limited-time interest-free delayed payment privilege; see, e.g., Khouja [6], Wang et al. [7], and references therein.

In modern consumer products market, numerous factors depending upon natural or business conditions affect various determinants of market demand. For example, the demand rate of a new type of air-conditioner, say air-conditioner A, is high in summer, which can be taken as a constant.
However, because of the seasons, the demand rate of airconditioner A will be lower after summer and a new type of air-conditioner will be launched into market the next year. Thus, the inventory system of air-conditioner A will have twostage demand; that is, the demand rate in the second stage is less than that in the first stage and which can be taken as a constant in the second stage.

Research considering the change in demand rate was widely studied. For a discrete case of time-varying demand pattern, Wagner and Whitin [8] established a dynamic programmimg model, and, for a time-proportional varying demand pattern, Resh et al. [9] proposed an optimal replenishment number and time scheduling stocking strategy. Donaldson [10] considered the inventory model in which the demand varies linearly with the time, and the model was extended by Dave $[11,12]$ to the case that the shortages are allowed. Dey et al. [13] and Muriana et al. [14], respectively, considered the inventory model with dynamic demand and stochastic demand. Different from the above, $\mathrm{Wu}$ et al. [15] derive the EOQ model for inventory of an item that deteriorates at a Weibull-distributed rate and assuming the demand rate with continuous function of time. Yan [16] considered the EOQ model with freshness-dependent demand for perishable items. Baker and Urban [17], Datta and Pal [18], and Urban [19] considered the inventory model 


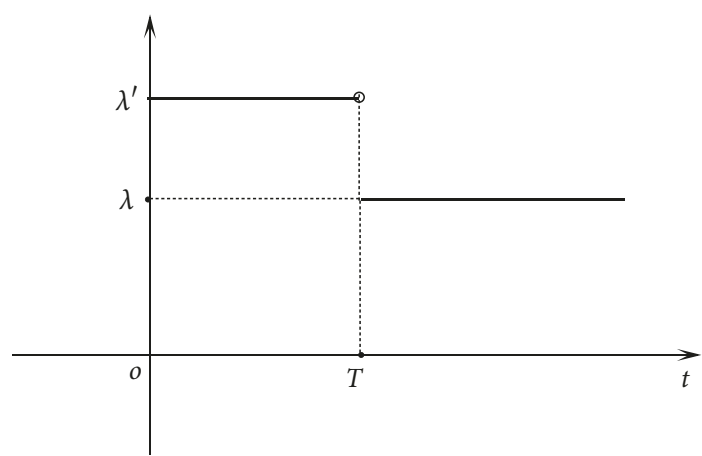

FIGURE 1: Two-stage demand inventory system with $\lambda^{\prime}>\lambda$.

TABLE 1: Notation.

\begin{tabular}{ll}
\hline symbol & description \\
\hline$\lambda^{\prime}$ & demand rate in the first stage \\
$\lambda$ & demand rate in the second stage \\
$K$ & retailer's fixed ordering cost \\
$h$ & holding cost per unit item per unit time \\
$p$ & backorder cost per unit item per unit time \\
$T$ & length of the first stage \\
\hline
\end{tabular}

wherein the demand depends on stock level, whereas Mandal and Phaujdar [20] considered the inventory model wherein the demand rate is linearly dependent on the inventory level and items are allowed to deteriorate over time.

In this paper, we consider the following extended version of the EOQ inventory model with step-shaped demand and backordering: the time horizon of the concerned inventory mechanism consists of two stages $[0, T]$ and $[T, \infty]$, and the demand rate in the first stage differs from that in the second stage; see Figure 1. Further, shortage is allowed and will be backordered. The other assumptions are the same as those on the EOQ model. For this mechanism, to minimize the retailer's inventory cost, we establish an optimization inventory decision model. By an inventory cost analysis, we present a closed-form solution to the model and provide an optimal replenishment and lot sizing strategy to the retailer. The given numerical experiments show the validity of the model.

The remainder of the paper is organized as follows. Section 2 presents the assumptions on the inventory model and notations used in the subsequent analysis. A mathematical formulation of the problem is also established in Section 3. In Section 4, we derive the optimal solution for the inventory model and provide an optimal replenishment policy to the retailer. Numerical experiments on sensitivity analysis to show the validity of the model are made in Section 5. The conclusion and some extensions are given in the last section.

\section{Notations and Assumptions}

First, we give the notations used in the subsequent analysis (Table 1) and then give the assumptions on the model.
Assumption 1. For the inventory system, we assume that

(1) the time horizon is infinite;

(2) the leading time for each order is zero;

(3) shortage is allowed and will be backordered;

(4) unit purchasing cost for both stages is the same;

(5) the fixed ordering and unit backordering costs for both stages are the same.

Without loss of generality, we also assume that the stock level is zero at the beginning inventory operation system.

\section{Problem Description and Model Formulation}

Consider the following inventory mechanism in which the time horizon of the concerned inventory mechanism consists of two stages $[0, T]$ and $[T, \infty]$, and the demand rate $\lambda^{\prime}$ in the first stage is strictly greater than $\lambda$ in the second stage; i.e., $\lambda^{\prime}>\lambda$. Further, shortage is allowed and will be backordered. The other assumptions are the same as those in the EOQ model; see Figure 1. For this inventory system, if the demand rate is constant, then the optimal ordering size at each replenishment circle is $Q^{\mathrm{BEOQ}}=\sqrt{2 \lambda K(h+p) / h p}$ with maximal backorder $B^{\mathrm{BEOQ}}=\sqrt{2 \lambda h K / p(h+p)}$ from the knowledge of inventory by Waters [5].

In our concerned inventory model, due to the skipping of the demand rate, to deduce the inventory cost, we should determine optimal order size and the maximal shortage level in the first stage so that the inventory cost is as lower as possible. As the inventory system would revert to the regular EOQ model with backordering, i.e., BEOQ model, once the replenishment cycles in the first stage are finished. To derive the optimal policy, we need the following "ideal" BEOQ ordering policy, abbreviated "IBEOQ": orders with size $Q_{\lambda^{\prime}}^{\mathrm{BEOQ}}=\sqrt{2 \lambda^{\prime} K(h+p) / h p}$ are made in the first stage and these ordering cycles are exactly ended at $T$, and orders with size $Q_{\lambda}^{\mathrm{BEOQ}}=\sqrt{2 \lambda K(h+p) / h p}$ are made in the second stage. Under this policy, the long-run average operating cost is $\sqrt{2 \lambda^{\prime} K h p /(h+p)}$ in the first stage and $\sqrt{2 \lambda K h p /(h+p)}$ in the second stage, respectively. On account of this, we may establish an optimal replenishment and stocking strategy model by minimizing the operating cost relative to the IBEOQ ordering policy.

For such an inventory system, as the demand rates of two stages are different, according to the inventory cost analysis, the retailer should make orders with different sizes in different stages. Suppose $m$ orders with sizes $Q_{1}, \ldots, Q_{m}$ are made in the first stage, and the maximal shortage levels are, respectively, $B_{1}, \ldots, B_{m}$ in each replenishment cycle. According to the inventory cost minimization principle, the first $(m-1)$ replenishment cycles would be ended before $T$ and the $m$-th replenishment cycle would be ended at or after $T$, i.e.,

$$
\begin{aligned}
Q_{1}+Q_{2}+\cdots+Q_{m-1}+B_{m-1} & <\lambda^{\prime} T, \\
Q_{1}+Q_{2}+\cdots+Q_{m}+B_{m} & \geq \lambda^{\prime} T .
\end{aligned}
$$


Further, once the $m$ replenishment cycles are ended, the system would resort to the BEOQ ordering policy with size $Q_{\lambda}^{B E O Q}=\sqrt{2 \lambda K(h+p) / h p}$. Thus, to minimize the retailer's inventory cost, we only need to determine the order sizes $Q_{1}, \ldots, Q_{m}$, and the maximum shortage levels $B_{1}, \ldots, B_{m}$. In this sense, we denote the operating cost for these replenishment cycles by $f\left(Q_{1}, \ldots, Q_{m}, B_{1}, \ldots, B_{m}\right)$, and denote the time horizon of these replenishment cycles by $T_{m}$. Since shortage will be fully backordered, it holds that

$$
T_{m}=T+\frac{\left(B_{m}+\sum_{i=1}^{m} Q_{i}-\lambda^{\prime} T\right)}{\lambda} .
$$

In the other way, since shortage is allowed and will be backordered, using the fact that the long-run average operating cost under the IBEOQ policy are $\sqrt{2 \lambda^{\prime} K h p /(h+p)}$ in the first stage and $\sqrt{2 \lambda K h p /(h+p)}$ in the second stage, we obtain the operating cost under the IBEOQ ordering policy over the time horizon $T_{m}$,

$$
\begin{aligned}
f_{\text {IBEOQ }}= & T \sqrt{\frac{2 \lambda^{\prime} K h p}{h+p}}+\left(T_{m}-T\right) \sqrt{\frac{2 \lambda K h p}{h+p}} \\
= & T \sqrt{\frac{2 \lambda^{\prime} K h p}{h+p}} \\
& +\sqrt{\frac{2 K h p}{\lambda(h+p)}}\left(B_{m}+\sum_{i=1}^{m} Q_{i}-\lambda^{\prime} T\right) .
\end{aligned}
$$

Accordingly, the operating cost under the ordering policy relative to the IBEOQ ordering policy over the time horizon $T_{m}$ is

$$
\begin{aligned}
& F\left(Q_{1}, \ldots, Q_{m}, B_{1}, \ldots, B_{m}\right) \\
& \quad=f\left(Q_{1}, \ldots, Q_{m}, B_{1}, \ldots, B_{m}\right)-f_{\text {IBEOQ }} .
\end{aligned}
$$

Then, the problem of determining an optimal policy can be formulated as the following optimization problem:

$$
\begin{array}{rl}
\min _{m, Q_{i}, B_{i}} & F\left(Q_{1}, \ldots, Q_{m}, B_{1}, \ldots, B_{m}\right) \\
\text { s.t. } & Q_{1}+Q_{2}+\cdots+Q_{m-1}+B_{m-1}<\lambda^{\prime} T \\
& Q_{1}+Q_{2}+\cdots+Q_{m}+B_{m} \geq \lambda^{\prime} T \\
& Q_{i}, B_{i}>0, \quad i=1, \ldots, m .
\end{array}
$$

In the subsequent sections, we will first compute the optimal ordering sizes and the maximum shortage levels for fixed ordering times in the first stage and then calculate the optimal ordering times in the first stage based on the inventory cost analysis. At the end, we present the algorithm for problem (5).

\section{Model Solution}

For the inventory system, if $m$ orders are made in the first stage with sizes $Q_{1}, \ldots, Q_{m}$, since shortages are allowed,

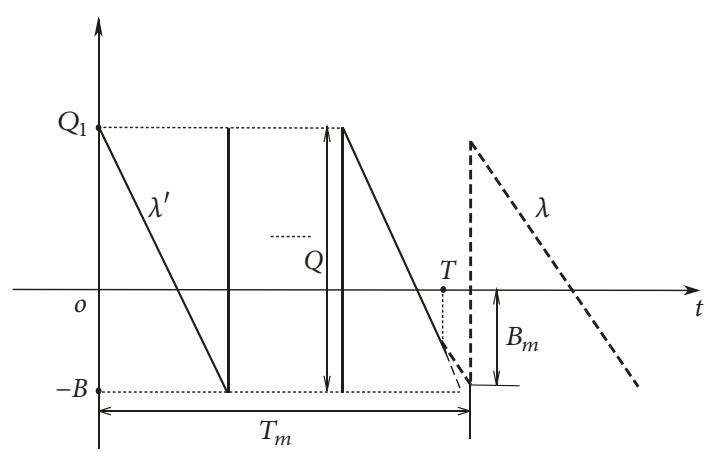

FIGURE 2: $\sum_{i=1}^{m} Q_{i}<\lambda^{\prime} T$.

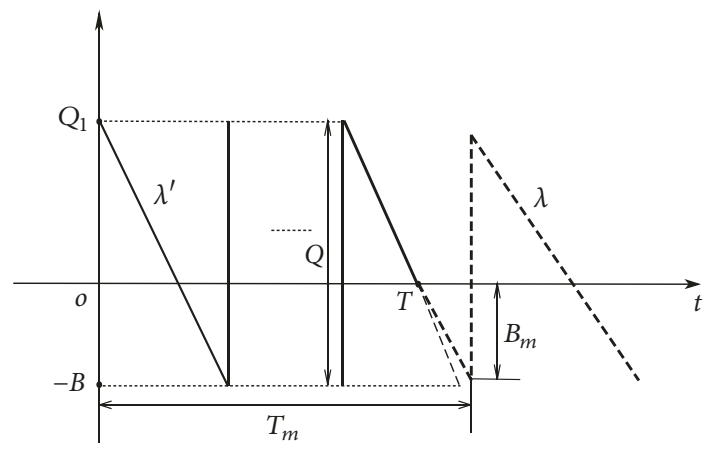

FIGURE 3: $\sum_{i=1}^{m} Q_{i}=\lambda^{\prime} T$.

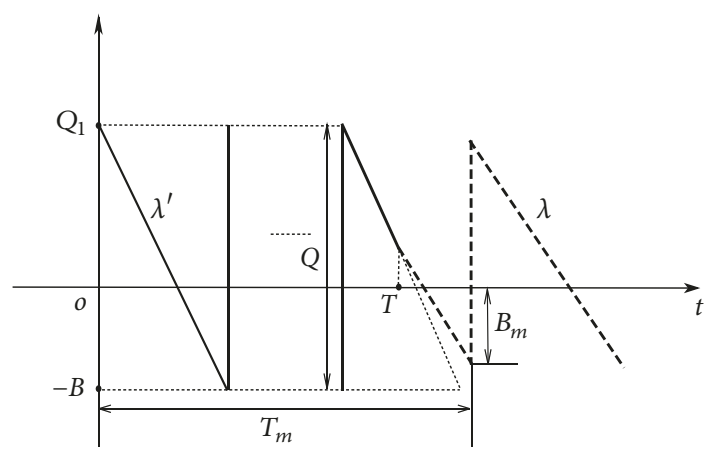

FigURE $4: \sum_{i=1}^{m} Q_{i}>\lambda^{\prime} T$.

according to the the size of the remaining stock $\sum_{i=1}^{m} Q_{i}-\lambda^{\prime} T$ at $T$, we have three scenarios: $\sum_{i=1}^{m} Q_{i}-\lambda^{\prime} T<0, \sum_{i=1}^{m} Q_{i}-$ $\lambda^{\prime} T=0$, and $\sum_{i=1}^{m} Q_{i}-\lambda^{\prime} T>0$. See Figures $2-4$.

4.1. Scenario $\sum_{i=1}^{m} Q_{i}<\lambda^{\prime} T$. This scenario is depicted in Figure 2, the stock position is exhausted and the shortage level is $\lambda^{\prime} T-\sum_{i=1}^{m} Q_{i}$ at $T$. From the knowledge of inventory, the total operating cost over the time length $T_{m}$ is

$$
\begin{aligned}
f\left(Q_{1}, \ldots, Q_{m}, B_{1}, \ldots, B_{m}\right) \\
=m K+\frac{h}{2 \lambda^{\prime}} Q_{1}^{2}+\frac{h}{2 \lambda^{\prime}} \sum_{i=2}^{m}\left(Q_{i}-B_{i-1}\right)^{2}+\frac{p}{2 \lambda^{\prime}} \sum_{i=1}^{m-1} B_{i}^{2} \\
+\frac{p}{2 \lambda} B_{m}^{2}+\left(\frac{p}{2 \lambda^{\prime}}-\frac{p}{2 \lambda}\right)\left(\lambda^{\prime} T-\sum_{i=1}^{m} Q_{i}\right)^{2} .
\end{aligned}
$$


Subsequently, substituting (6) and (3) into (4) yields that

$$
\begin{aligned}
& F\left(Q_{1}, \ldots, Q_{m}, B_{1}, \ldots, B_{m}\right) \\
& =m K+\frac{h}{2 \lambda^{\prime}} Q_{1}^{2}+\frac{h}{2 \lambda^{\prime}} \sum_{i=2}^{m}\left(Q_{i}-B_{i-1}\right)^{2}+\frac{p}{2 \lambda^{\prime}} \sum_{i=1}^{m-1} B_{i}^{2} \\
& +\frac{p}{2 \lambda} B_{m}^{2}+\left(\frac{p}{2 \lambda^{\prime}}-\frac{p}{2 \lambda}\right)\left(\lambda^{\prime} T-\sum_{i=1}^{m} Q_{i}\right)^{2} \\
& -\sqrt{\frac{2 K h p}{\lambda(h+p)}}\left(B_{m}+\sum_{i=1}^{m} Q_{i}\right)-T \sqrt{\frac{2 \lambda^{\prime} K h p}{h+p}} \\
& +\lambda^{\prime} T \sqrt{\frac{2 K h p}{\lambda(h+p)}} .
\end{aligned}
$$

Then, the problem (5) can be formulated as the following optimization problem:

$$
\begin{array}{rl}
\min _{m, Q_{i}, B_{i}} & F\left(Q_{1}, \ldots, Q_{m}, B_{1}, \ldots, B_{m}\right) \\
\text { s.t. } & Q_{1}+Q_{2}+\cdots+Q_{m-1}+B_{m-1}<\lambda^{\prime} T \\
& Q_{1}+Q_{2}+\cdots+Q_{m}<\lambda^{\prime} T \\
& Q_{1}+Q_{2}+\cdots+Q_{m}+B_{m} \geq \lambda^{\prime} T \\
& Q_{i}, B_{i}>0, \quad i=1, \ldots, m
\end{array}
$$

For this problem, we have the following conclusion.

Theorem 1. For the inventory model (8), suppose $m$ orders are made in the first stage. If $m \leq \min \left\{u_{1}, u_{2}\right\}$, then the optimal ordering sizes and the optimal maximum shortage level in the first stage are, respectively,

$$
\begin{aligned}
Q_{1} & =\frac{p \lambda^{\prime} T}{(h+p) m}, \\
Q_{i} & =\frac{\lambda^{\prime} T}{m}, \quad i=2,3, \ldots, m, \\
B_{i} & =\frac{h \lambda^{\prime} T}{(h+p) m}, \quad i=1,2, \ldots, m
\end{aligned}
$$

and if $\min \left\{u_{1}, u_{2}\right\}<m<\max \left\{u_{1}, u_{2}\right\}$, then the optimal ordering sizes and the optimal maximum shortage level in the first stage are, respectively,

$$
\begin{aligned}
Q_{1} & =\frac{\lambda^{\prime}(\beta-\alpha p T)}{(h+p)\left(h \lambda^{\prime}-\alpha m\right)}, \\
Q_{i} & =\frac{\lambda^{\prime}(\beta-\alpha p T)}{p\left(h \lambda^{\prime}-\alpha m\right)}, \quad i=2,3, \ldots, m, \\
B_{i} & =\frac{h \lambda^{\prime}(\beta-\alpha p T)}{p(h+p)\left(h \lambda^{\prime}-\alpha m\right)}, \quad i=1,2, \ldots, m-1 \\
B_{m} & =\sqrt{\frac{2 \lambda K h}{p(h+p)}}
\end{aligned}
$$

where $u_{1}=\lambda^{\prime} T \sqrt{h p / 2 \lambda K(h+p)}, u_{2}=T \sqrt{\lambda h p / 2 K(h+p)}+$ $h /(h+p), \alpha=(h+p)\left(\lambda^{\prime}-\lambda\right), \beta=\sqrt{2 \lambda K h p(h+p)}$.

Proof. For problem (8), since the constraints are linear, any optimal solution is a KKT point which satisfies one of the following two systems. See Wang et al. [21, 22].

(1) System 1:

$$
\begin{aligned}
& \frac{\partial F}{\partial Q_{1}} \\
& =\frac{h}{\lambda^{\prime}} Q_{1}-\left(\frac{p}{\lambda^{\prime}}-\frac{p}{\lambda}\right)\left(\lambda^{\prime} T-\sum_{i=1}^{m} Q_{i}\right) \\
& \\
& \quad-\sqrt{\frac{2 K h p}{\lambda(h+p)}}=\mu,
\end{aligned}
$$

$\frac{\partial F}{\partial Q_{i}}$

$$
\begin{aligned}
= & \frac{h}{\lambda^{\prime}}\left(Q_{i}-B_{i-1}\right)-\left(\frac{p}{\lambda^{\prime}}-\frac{p}{\lambda}\right)\left(\lambda^{\prime} T-\sum_{i=1}^{m} Q_{i}\right) \\
& -\sqrt{\frac{2 K h p}{\lambda(h+p)}}=\mu, \quad i=2, \ldots, m, \\
\frac{\partial F}{\partial B_{i}}= & -\frac{h}{\lambda^{\prime}}\left(Q_{i+1}-B_{i}\right)+\frac{p}{\lambda^{\prime}} B_{i}=0, \quad i=1, \ldots, m-1,
\end{aligned}
$$

$$
\begin{aligned}
& \frac{\partial F}{\partial B_{m}}=\frac{p}{\lambda} B_{m}-\sqrt{\frac{2 K h p}{\lambda(h+p)}}=\mu, \\
& Q_{1}+Q_{2}+\cdots+Q_{m-1}+B_{m-1}<\lambda^{\prime} T, \\
& Q_{1}+Q_{2}+\cdots+Q_{m}<\lambda^{\prime} T, \\
& Q_{1}+Q_{2}+\cdots+Q_{m}+B_{m}=\lambda^{\prime} T, \\
& Q_{i}, B_{i}>0, \quad i=1, \ldots, m,
\end{aligned}
$$

where $\mu \geq 0$ is the Lagrange multiplier corresponding with the equality constraint.

(2) System 2:

$$
\begin{aligned}
& \frac{\partial F}{\partial Q_{1}} \\
& \quad=\frac{h}{\lambda^{\prime}} Q_{1}-\left(\frac{p}{\lambda^{\prime}}-\frac{p}{\lambda}\right)\left(\lambda^{\prime} T-\sum_{i=1}^{m} Q_{i}\right) \\
& \quad-\sqrt{\frac{2 K h p}{\lambda(h+p)}}=0, \\
& \frac{\partial F}{\partial Q_{i}} \quad \\
& =\frac{h}{\lambda^{\prime}}\left(Q_{i}-B_{i-1}\right)-\left(\frac{p}{\lambda^{\prime}}-\frac{p}{\lambda}\right)\left(\lambda^{\prime} T-\sum_{i=1}^{m} Q_{i}\right)
\end{aligned}
$$




$$
\begin{aligned}
& -\sqrt{\frac{2 K h p}{\lambda(h+p)}}=0, \quad i=2, \ldots, m, \\
& \frac{\partial F}{\partial B_{i}}=-\frac{h}{\lambda^{\prime}}\left(Q_{i+1}-B_{i}\right)+\frac{p}{\lambda^{\prime}} B_{i}=0, \\
& \frac{\partial F}{\partial B_{m}}=\frac{p}{\lambda} B_{m}-\sqrt{\frac{2 K h p}{\lambda(h+p)}}=0, \\
& Q_{1}+Q_{2}+\cdots+Q_{m-1}+B_{m-1}<\lambda^{\prime} T, \\
& Q_{1}+Q_{2}+\cdots+Q_{m}<\lambda^{\prime} T, \\
& Q_{1}+Q_{2}+\cdots+Q_{m}+B_{m}>\lambda^{\prime} T, \\
& Q_{i}, B_{i}>0, \quad i=1, \ldots, m
\end{aligned}
$$

For these two systems, using their former $(2 m-1)$ equations, respectively, we can all conclude that

$$
\begin{aligned}
& Q_{2}=\cdots=Q_{m}, \\
& B_{1}=\cdots=B_{m-1}=\frac{h}{h+p} Q_{i}, \\
& Q_{1}=\frac{p}{h+p} Q_{i}, \quad i=2, \ldots, m .
\end{aligned}
$$

For the former system, substituting (13) into the last equation and combining the other equations yield

$$
\begin{aligned}
Q_{1} & =\frac{p \lambda^{\prime} T}{(h+p) m}, \\
Q_{i} & =\frac{\lambda^{\prime} T}{m}, \quad i=2,3, \ldots, m, \\
B_{i} & =\frac{h \lambda^{\prime} T}{(h+p) m}, \quad i=1,2, \ldots, m
\end{aligned}
$$

with $\mu=p h \lambda^{\prime} T / m \lambda(h+p)-\sqrt{2 K h p / \lambda(h+p)}$. It is easy to verify that all the inequalities of the former system hold for $Q_{i}$, and $B_{i}$ given above. In addition, to guarantee $Q_{i}$ and $B_{i}$ given above constitute a KKT point of problem (8), $\mu \geq 0$ is required; that is,

$$
m \leq \lambda^{\prime} T \sqrt{\frac{p h}{2 \lambda K(h+p)}}=u_{1} .
$$

So the equation set by (14) is the solution to the problem (8) provided that

$$
m \leq u_{1} .
$$

For the latter system, after some substitutions and algebra, we have: if $m(h+p)\left(\lambda^{\prime}-\lambda\right) \neq \lambda^{\prime} h$, then

$$
\begin{aligned}
Q_{1}= & \frac{p}{h+p} \\
& \cdot \frac{\lambda^{\prime}\left[\sqrt{2 \lambda K h p(h+p)}-p T(h+p)\left(\lambda^{\prime}-\lambda\right)\right]}{p\left[h \lambda^{\prime}-m(h+p)\left(\lambda^{\prime}-\lambda\right)\right]}, \\
Q_{i}= & \frac{\lambda^{\prime}\left[\sqrt{2 \lambda K h p(h+p)}-p T(h+p)\left(\lambda^{\prime}-\lambda\right)\right]}{p\left[h \lambda^{\prime}-m(h+p)\left(\lambda^{\prime}-\lambda\right)\right]}, \\
B_{i}= & \frac{h}{h+p} \quad i=2, \ldots, m, \\
& \frac{\lambda^{\prime}\left[\sqrt{2 \lambda K h p(h+p)}-p T(h+p)\left(\lambda^{\prime}-\lambda\right)\right]}{p\left[h \lambda^{\prime}-m(h+p)\left(\lambda^{\prime}-\lambda\right)\right]}, \\
B_{m}= & \sqrt{\frac{2 \lambda K h}{p(h+p)}} .
\end{aligned}
$$

otherwise, the equations of the latter system have no solution.

Denote

$$
\begin{aligned}
& u_{1}=\lambda^{\prime} T \sqrt{\frac{h p}{2 \lambda K(h+p)}}, \\
& u_{2}=T \sqrt{\frac{\lambda h p}{2 K(h+p)}}+\frac{h}{h+p}, \\
& \alpha=(h+p)\left(\lambda^{\prime}-\lambda\right), \\
& \beta=\sqrt{2 \lambda K h p(h+p)} .
\end{aligned}
$$

To guarantee $Q_{i}$ and $B_{i}$ given by (17) is a KKT point of problem (8), we break up the discussion into two cases.

Case $1\left(\sqrt{2 \lambda K h p(h+p)} \geq p T(h+p)\left(\lambda^{\prime}-\lambda\right)\right.$, i.e., $\left.\beta \geq \alpha p T\right)$. In this case, to guarantee the nonnegativity of the $Q_{i}$ and $B_{i}$, it needs that $m(h+p)\left(\lambda^{\prime}-\lambda\right)<\lambda^{\prime} h$, i.e., $m<\lambda^{\prime} h /(h+p)\left(\lambda^{\prime}-\right.$ $\lambda)$. Substituting (17) into the second inequality and the last inequality in the latter system yields that

$$
\lambda^{\prime} T \sqrt{\frac{p h}{2 \lambda K(h+p)}}<m<T \sqrt{\frac{\lambda h p}{2 K(h+p)}}+\frac{h}{h+p} .
$$

Moreover, under this case, we have

$$
\begin{aligned}
\lambda^{\prime} T \sqrt{\frac{p h}{2 \lambda K(h+p)}} & <T \sqrt{\frac{\lambda h p}{2 K(h+p)}}+\frac{h}{h+p} \\
& <\frac{\lambda^{\prime} h}{(h+p)\left(\lambda^{\prime}-\lambda\right)},
\end{aligned}
$$


so $Q_{i}$ and $B_{i}$ given by (17) are a solution to the latter system provided that

$$
\begin{gathered}
\beta \geq \alpha p T, \\
u_{1}<m<u_{2} .
\end{gathered}
$$

Case $2(\beta<\alpha p T)$. By a similar discussion to that for Case 1 , we know that $Q_{i}$ and $B_{i}$ given by (17) are a solution to the latter system provided that

$$
\begin{gathered}
\beta<\alpha p T, \\
u_{2}<m<u_{1} .
\end{gathered}
$$

Substituting (14) and (17) into (8), respectively, yields that the operating cost over the interval of length $T_{m}$ under the two policies relative to the IBEOQ ordering policy are $F_{\mathrm{I}}$ and $F_{\mathrm{II}}$, respectively.

$$
\begin{aligned}
F_{\mathrm{I}}= & m K+\frac{h p \lambda^{\prime} T^{2}}{2 m(h+p)}-T \sqrt{\frac{2 \lambda^{\prime} K h p}{h+p}}, \\
F_{\mathrm{II}}= & m K+\frac{h \lambda^{\prime}[m(h+p)-h](\beta-\alpha p T)^{2}}{2 p(h+p)^{2}\left(\lambda^{\prime} h-m \alpha\right)^{2}} \\
& +\frac{\lambda^{\prime}\left(\lambda-\lambda^{\prime}\right)[(h+p) \beta m-(h \beta+\gamma)]^{2}}{2 \lambda p(h+p)^{2}\left(\lambda^{\prime} h-m \alpha\right)^{2}} \\
& -\frac{\lambda^{\prime} \beta[m(h+p)-h](\beta-\alpha p T)}{\lambda p(h+p)^{2}\left(\lambda^{\prime} h-m \alpha\right)}-\frac{K h}{h+p} \\
& +\lambda^{\prime} T \sqrt{\frac{2 K h p}{\lambda(h+p)}}-T \sqrt{\frac{2 \lambda^{\prime} K h p}{h+p}},
\end{aligned}
$$

where $\gamma=\lambda h p T(h+p)$.

Under the Case 2, if $u_{2}<m<u_{1}$, after some substitutions and algebra, we can prove that $F_{\mathrm{I}}(m)>F_{\mathrm{II}}(m)$.

Combining the discussions above, we conclude that $Q, B$, and $B_{m}$ given by (14) are a solution of problem (8) provided that

$$
m \leq \min \left\{u_{1}, u_{2}\right\}
$$

and (17) is a solution of problem (8) provided that

$$
\min \left\{u_{1}, u_{2}\right\}<m<\max \left\{u_{1}, u_{2}\right\} .
$$

The proof is completed by combining the discussions above.

By Theorem 1, we know that the optimal ordering sizes from second order to $\mathrm{m}$-th order in the first stage are the same, and the optimal maximum shortage levels from the first order to (m-1)-th order in the first stage are the same too; i.e.,

$$
\begin{aligned}
& Q_{2}=\cdots=Q_{m} \triangleq Q, \\
& B_{1}=\cdots=B_{m-1} \triangleq B .
\end{aligned}
$$

also

$$
\begin{aligned}
B & =\frac{h}{h+p} Q, \\
Q_{1} & =\frac{p}{h+p} Q .
\end{aligned}
$$

So we can obtain the following two ordering policies for problem (5) with $m$ orders in the first stage.

Policy $\pi_{\mathrm{I}}$

$$
\begin{aligned}
Q & =\frac{\lambda^{\prime} T}{m}, \\
Q_{1} & =\frac{p}{h+p} Q, \\
B & =B_{m}=\frac{h}{h+p} Q,
\end{aligned}
$$

which applies to the case that $m \leq \min \left\{u_{1}, u_{2}\right\}$.

Policy $_{\mathrm{II}}$

$$
\begin{aligned}
Q & =\frac{\lambda^{\prime}(\beta-\alpha p T)}{p\left(h \lambda^{\prime}-m \alpha\right)}, \\
Q_{1} & =\frac{p}{h+p} Q, \\
B & =\frac{h}{h+p} Q, \\
B_{m} & =\sqrt{\frac{2 \lambda K h}{p(h+p)}},
\end{aligned}
$$

which applies to the case that $\min \left\{u_{1}, u_{2}\right\}<m<$ $\max \left\{u_{1}, u_{2}\right\}$.

Now, we consider the optimal ordering times in the first stage under Policy $\pi_{\mathrm{I}}$ and Policy $\pi_{\mathrm{II}}$.

By the proof process of Theorem 1, we know that the operating costs over the interval of length $T_{m}$ under the two policies relative to the IBEOQ ordering policy are $F_{\mathrm{I}}$ and $F_{\mathrm{II}}$, respectively.

For function $F_{\mathrm{I}}$, by

$$
F_{\text {I }}^{\prime \prime}(m)=\frac{h p \lambda^{\prime} T^{2}}{(h+p) m^{3}}>0,
$$

we know that $F_{\mathrm{I}}(m)$ is a convex function in $m$, and its minimum can be obtained at the stationary point of the function; i.e.,

$$
\bar{m}_{\mathrm{I}}=T \sqrt{\frac{\lambda^{\prime} h p}{2 K(h+p)}} .
$$

Set $m_{\mathrm{I}_{1}}=\left\lfloor\bar{m}_{\mathrm{I}}\right\rfloor, m_{\mathrm{I}_{2}}=\left\lceil\bar{m}_{\mathrm{I}}\right\rceil, m_{\mathrm{I}_{3}}=\left\lfloor\min \left\{u_{1}, u_{2}\right\}\right\rfloor$. Then due to the fact that $m$ is a positive integer, and requires $m \leq$ $\min \left\{u_{1}, u_{2}\right\}$. So the optimal ordering times in the first stage 
are $m_{\mathrm{I}}=m_{\mathrm{I}_{1}}$ provided that $m_{\mathrm{I}_{1}} \leq \min \left\{u_{1}, u_{2}\right\}$ and $F_{\mathrm{I}}\left(m_{\mathrm{I}_{1}}\right) \leq$ $F_{\mathrm{I}}\left(m_{\mathrm{I}_{2}}\right)$ or $m_{\mathrm{I}_{2}}>\min \left\{u_{1}, u_{2}\right\}$, or the optimal ordering times are $m_{\mathrm{I}}=m_{\mathrm{I}_{2}}$ provided that $m_{\mathrm{I}_{2}} \leq \min \left\{u_{1}, u_{2}\right\}$, and $F_{\mathrm{I}}\left(m_{\mathrm{I}_{1}}\right)>$
$F_{\mathrm{I}}\left(m_{\mathrm{I}_{2}}\right)$. Moreover, if $m_{\mathrm{I}_{1}}>\min \left\{u_{1}, u_{2}\right\}$, the optimal ordering times in the first stage are $m_{\mathrm{I}}=m_{\mathrm{I}_{3}}$. That is

$$
m^{*}= \begin{cases}m_{\mathrm{I}_{1}} & \text { if } m_{\mathrm{I}_{1}} \leq \min \left\{u_{1}, u_{2}\right\} \text { and } F_{\mathrm{I}}\left(m_{\mathrm{I}_{1}}\right) \leq F_{\mathrm{I}}\left(m_{\mathrm{I}_{2}}\right) \text { or } m_{\mathrm{I}_{2}}>\min \left\{u_{1}, u_{2}\right\}, \\ m_{\mathrm{I}_{2}} & \text { if } m_{\mathrm{I}_{2}} \leq \min \left\{u_{1}, u_{2}\right\} \text { and } F_{\mathrm{I}}\left(m_{\mathrm{I}_{1}}\right)>F_{\mathrm{I}}\left(m_{\mathrm{I}_{2}}\right), \\ m_{\mathrm{I}_{3}} & \text { if } m_{\mathrm{I}_{1}}>\min \left\{u_{1}, u_{2}\right\} .\end{cases}
$$

For function $F_{\text {II }}$ given by (24), by

$$
F_{\text {II }}^{\prime \prime}(m)=-\frac{\alpha \lambda^{\prime} h(\beta-\alpha p T)^{2}}{p(h+p)\left(\lambda^{\prime} h-m \alpha\right)^{3}}
$$

we know that if $\lambda^{\prime} h-m \alpha<0$, i.e., $m>\lambda^{\prime} h / \alpha$, then $F_{\text {II }}^{\prime \prime}(m)>0$ and $F_{\mathrm{II}}(m)$ is a convex function in $m$, and its minimum can be obtained at the stationary point of the function; i.e.,

$$
\bar{m}_{\mathrm{II}}=\frac{h \lambda^{\prime}}{\alpha}+\sqrt{\frac{\lambda^{\prime} h}{2 K p(h+p)}}\left|\frac{\beta}{\alpha}-p T\right|>\frac{h \lambda^{\prime}}{\alpha} .
$$

On the other hand, according to the proof of Theorem 1 , if $m>\lambda^{\prime} h / \alpha$, to guarantee $Q_{i}, B_{i}$, and $B_{m}$ given by (17) is a solution to the model (8)), it needs that

$$
\begin{gathered}
\beta<\alpha p T, \\
u_{2}<m<u_{1} .
\end{gathered}
$$

In this case, the optimal ordering times in the first stage are

$$
\begin{aligned}
\bar{m}_{\mathrm{II}} & =\frac{h \lambda^{\prime}}{\alpha}+\sqrt{\frac{\lambda^{\prime} h}{2 K p(h+p)}}\left(p T-\frac{\beta}{\alpha}\right) \\
& =T \sqrt{\frac{\lambda^{\prime} h p}{2 K(h+p)}+\frac{h\left(\lambda^{\prime}-\sqrt{\lambda \lambda^{\prime}}\right)}{\left(\lambda^{\prime}-\lambda\right)(h+p)}},
\end{aligned}
$$

which satisfies that $u_{2}<\overline{m_{\mathrm{II}}}<u_{1}$.

However, if $\lambda^{\prime} h-m \alpha>0$, i.e., $m<\lambda^{\prime} h / \alpha$, then $F_{\text {II }}^{\prime \prime}(m)<0$ and $F_{\text {II }}(m)$ is a concave function in $m$, and its minimum can be obtained at the terminal point $\left(u_{1}\right.$ and $\left.u_{2}\right)$ of the function, and, according to the proof of Theorem 1, under the case that

$$
\begin{gathered}
\beta \geq \alpha p T, \\
u_{1}<m<u_{2} .
\end{gathered}
$$

Denote $m_{\mathrm{II}_{1}}=\left\lfloor\bar{m}_{\mathrm{II}}\right\rfloor, m_{\mathrm{II}_{2}}=\left\lceil\bar{m}_{\mathrm{II}}\right\rceil, m_{\mathrm{II}_{3}}=\left\lceil u_{1}\right\rceil . m_{\mathrm{II}_{4}}=$ $\left\lfloor u_{2}\right\rfloor$. Obviously, $m_{\mathrm{II}_{1}}<u_{1}, m_{\mathrm{II}_{2}}>u_{2}, m_{\mathrm{II}_{3}}>u_{1}, m_{\mathrm{II}_{4}}<$ $u_{2}$. Based on the discussion above, we obtain the optimal ordering times in the first stage under Policy $\pi_{\mathrm{II}}$

$$
m^{*}= \begin{cases}m_{\mathrm{II}_{1}}, & \text { if } \beta<\alpha p T, u_{2}<m_{\mathrm{II}_{1}} \leq m_{\mathrm{II}_{2}}<u_{1}, F_{\mathrm{II}}\left(m_{\mathrm{II}_{1}}\right) \leq F_{\mathrm{II}}\left(m_{\mathrm{II}_{2}}\right), \\ & \text { or } \beta<\alpha p T, u_{2}<m_{\mathrm{II}_{1}}<u_{1} \leq m_{\mathrm{II}_{2}} ; \\ m_{\mathrm{II}_{2}}, & \text { if } \beta<\alpha p T, u_{2}<m_{\mathrm{II}_{1}} \leq m_{\mathrm{II}_{2}}<u_{1}, F_{\mathrm{II}}\left(m_{\mathrm{II}_{1}}\right)>F_{\mathrm{II}}\left(m_{\mathrm{II}_{2}}\right) \\ & \text { or } \beta<\alpha p T, m_{\mathrm{II}_{1}} \leq u_{2}<m_{\mathrm{II}_{2}}<u_{1} ; \\ m_{\mathrm{II}_{3}}, & \text { if } \beta \geq \alpha p T, m_{\mathrm{II}_{3}}<u_{2}, m_{\mathrm{II}_{4}}>u_{1}, F_{\mathrm{II}}\left(m_{\mathrm{II}_{3}}\right) \leq F_{\mathrm{II}}\left(m_{\mathrm{II}_{4}}\right), \\ & \beta \geq \alpha p T, m_{\mathrm{II}_{4}} \leq u_{1}<m_{\mathrm{II}_{3}}<u_{2} ; \\ m_{\mathrm{II}_{4}}, & \text { if } \beta \geq \alpha p T, m_{\mathrm{II}_{3}}<u_{2}, m_{\mathrm{II}_{4}}>u_{1}, F_{\mathrm{II}}\left(m_{\mathrm{II}_{3}}\right)>F_{\mathrm{II}}\left(m_{\mathrm{II}_{4}}\right), \\ & \text { or } \beta \geq \alpha p T, u_{1}<m_{\mathrm{II}_{4}}<u_{2} \leq m_{\mathrm{II}_{3}} .\end{cases}
$$

4.2. Scenario $\sum_{i=1}^{m} Q_{i}=\lambda^{\prime} T$. In this scenario, $m$ orders are made in the first stage and they can exactly meet the demand of the first stage; see Figure 3. Obviously, $T_{m}=T+B_{m} / \lambda$. From the knowledge of inventory, we have

$$
f\left(Q_{1}, \ldots, Q_{m}, B_{1}, \ldots, B_{m}\right)
$$

$$
\begin{aligned}
= & m K+\frac{h}{2 \lambda^{\prime}} Q_{1}^{2}+\frac{h}{2 \lambda^{\prime}} \sum_{i=2}^{m}\left(Q_{i}-B_{i-1}\right)^{2}+\frac{p}{2 \lambda^{\prime}} \sum_{i=1}^{m-1} B_{i}^{2} \\
& +\frac{p}{2 \lambda} B_{m}^{2}
\end{aligned}
$$




$$
f_{I B E O Q}=T \sqrt{\frac{2 \lambda^{\prime} K h p}{h+p}}+B_{m} \sqrt{\frac{2 K h p}{\lambda(h+p)}} .
$$

Substituting (40) and (41) into formula (4) yields that

$$
\begin{gathered}
F\left(Q_{1}, \ldots, Q_{m}, B_{1}, \ldots, B_{m}\right) \\
=m K+\frac{h}{2 \lambda^{\prime}} Q_{1}^{2}+\frac{h}{2 \lambda^{\prime}} \sum_{i=2}^{m}\left(Q_{i}-B_{i-1}\right)^{2}+\frac{p}{2 \lambda^{\prime}} \sum_{i=1}^{m-1} B_{i}^{2} \\
+\frac{p}{2 \lambda} B_{m}^{2}-B_{m} \sqrt{\frac{2 K h p}{\lambda(h+p)}}-T \sqrt{\frac{2 \lambda^{\prime} K h p}{h+p}}
\end{gathered}
$$

Then problem (5) can be formulated as the following optimization problem:

$$
\begin{array}{rl}
\min _{m, Q_{i}, B_{i}} & F\left(Q_{1}, \ldots, Q_{m}, B_{1}, \ldots, B_{m}\right) \\
\text { s.t. } & Q_{1}+Q_{2}+\cdots+Q_{m}=\lambda^{\prime} T \\
& Q_{i}, B_{i}>0, \quad i=1, \ldots, m .
\end{array}
$$

For this problem, we have the following conclusion.

Theorem 2. For inventory model (43), suppose $m$ orders are made in the first stage; then the optimal ordering sizes and the optimal maximum shortage level in the first stage are, respectively,

$$
\begin{aligned}
Q_{1} & =\frac{p \lambda^{\prime} T}{m(h+p)-h}, \\
Q_{i} & =\frac{(h+p) \lambda^{\prime} T}{m(h+p)-h}, \quad i=2,3, \ldots, m \\
B_{i} & =\frac{h \lambda^{\prime} T}{m(h+p)-h}, \quad i=1,2 \cdots, m-1 \\
B_{m} & =\sqrt{\frac{2 \lambda K h}{p(h+p)}} .
\end{aligned}
$$

Proof. For problem (43), its KKT point satisfies the following system:

$$
\begin{aligned}
& \frac{\partial F}{\partial Q_{1}}=\frac{h}{\lambda^{\prime}} Q_{1}=\mu, \\
& \frac{\partial F}{\partial Q_{i}}=\frac{h}{\lambda^{\prime}}\left(Q_{i}-B_{i-1}\right)=\mu,
\end{aligned}
$$$$
i=2, \ldots, m \text {, }
$$

$$
\begin{aligned}
\frac{\partial F}{\partial B_{i}} & =-\frac{h}{\lambda^{\prime}}\left(Q_{i+1}-B_{i}\right)+\frac{p}{\lambda^{\prime}} B_{i}=0, \\
i=1, \ldots, m-1, & \\
\frac{\partial F}{\partial B_{m}} & =\frac{p}{\lambda} B_{m}-\sqrt{\frac{2 K h p}{\lambda(h+p)}}=0, \\
Q_{1}+Q_{2}+\cdots+Q_{m} & =\lambda^{\prime} T, \\
Q_{i}, B_{i} & \geq 0, \quad i=1, \ldots, m .
\end{aligned}
$$

where $\mu \geq 0$ is the optimal Lagrange multiplier.

By solving the equations in the system, we can obtain the desired conclusion.

For this scenario, by Theorem 2, the same as Scenario 1, the optimal ordering sizes \{denoted as $Q$ \} from second order to $\mathrm{m}$-th order in the first stage are the same, and the optimal maximum shortage levels \{denoted as $B$ \} from the first order to (m-1)-th order in the first stage are the same too, so we can obtain the following optimal ordering policy for problem (5):

Policy $_{\mathrm{III}}$

$$
Q=\frac{(h+p) \lambda^{\prime} T}{m(h+p)-h}
$$

$$
\begin{aligned}
Q_{1} & =\frac{p}{h+p} Q, \\
B & =\frac{h}{h+p} Q, \\
B_{m} & =\sqrt{\frac{2 \lambda K h}{p(h+p)}} .
\end{aligned}
$$

Under this policy, the operating cost over the interval of length $T_{m}$ relative to the IBEOQ ordering policy is

$$
\begin{aligned}
F_{\mathrm{III}}= & m K+\frac{h p \lambda^{\prime} T^{2}}{2[m(h+p)-h]}-\frac{K h}{h+p} \\
& -T \sqrt{\frac{2 \lambda^{\prime} K h p}{h+p}} .
\end{aligned}
$$

Now, we consider the optimal ordering times in the first stage under Policies $\pi_{\mathrm{III}}$.

For function $F_{\mathrm{III}}$, by

$$
F_{\text {III }}^{\prime \prime}(m)=\frac{h p(h+p)^{2}}{[m(h+p)-h]^{3}} \lambda^{\prime} T^{2}>0,
$$

we know that $F_{\mathrm{III}}(m)$ is a convex function in $m$, and its minimum can be obtained at the stationary point of the function, i.e.,

$$
F_{\mathrm{III}}^{\prime}(\mathrm{m})=K-\frac{h p(h+p)}{2[m(h+p)-h]} \lambda^{\prime} T^{2}=0 .
$$


Set

$$
\begin{aligned}
& \bar{m}_{\mathrm{III}}=T \sqrt{\frac{\lambda^{\prime} h p}{2 K(h+p)}}+\frac{h}{h+p}, \\
& m_{\mathrm{III}_{1}}=\left\lfloor\bar{m}_{\mathrm{III}}\right\rfloor, \\
& m_{\mathrm{III}_{2}}=\left\lceil\bar{m}_{\mathrm{III}}\right\rceil .
\end{aligned}
$$

Then, the optimal ordering times in the first stage are $m_{\text {III }}=$ $m_{\mathrm{III}_{1}}$ provided that $F_{\mathrm{III}}\left(m_{\mathrm{III}}\right) \leq F_{\mathrm{III}}\left(m_{\mathrm{III}_{2}}\right)$, and $m_{\mathrm{III}}=m_{\mathrm{III}_{2}}$ otherwise. Then, the optimal order times under Policy $\pi_{\mathrm{III}}$ are that

$$
m^{*}= \begin{cases}m_{\mathrm{III}_{1}} & \text { if } F_{\mathrm{III}}\left(m_{\mathrm{III}}\right) \leq F_{\mathrm{III}}\left(m_{\mathrm{II}_{2}}\right), \\ m_{\mathrm{III}_{2}} & \text { if } F_{\mathrm{III}}\left(m_{\mathrm{III}_{1}}\right)>F_{\mathrm{III}}\left(m_{\mathrm{III}_{2}}\right) .\end{cases}
$$

4.3. Scenario $\sum_{i=1}^{m} Q_{i}>\lambda^{\prime} T$. For this scenario, see Figure 4; a similar discussions to that for Scenario 1 yields

$$
\begin{aligned}
f\left(Q_{1}, \ldots, Q_{m}, B_{1}, \ldots, B_{m}\right) \\
=m K+\frac{h}{2 \lambda^{\prime}} Q_{1}^{2}+\frac{h}{2 \lambda^{\prime}} \sum_{i=2}^{m}\left(Q_{i}-B_{i-1}\right)^{2}+\frac{p}{2 \lambda^{\prime}} \sum_{i=1}^{m-1} B_{i}^{2} \\
\quad+\frac{p}{2 \lambda} B_{m}^{2}+\left(\frac{h}{2 \lambda}-\frac{h}{2 \lambda^{\prime}}\right)\left(\sum_{i=1}^{m} Q_{i}-\lambda^{\prime} T\right)^{2} .
\end{aligned}
$$

So

$$
\begin{aligned}
& F\left(Q_{1}, \ldots, Q_{m}, B_{1}, \ldots, B_{m}\right) \\
&= m K+\frac{h}{2 \lambda^{\prime}} Q_{1}^{2}+\frac{h}{2 \lambda^{\prime}} \sum_{i=2}^{m}\left(Q_{i}-B_{i-1}\right)^{2}+\frac{p}{2 \lambda^{\prime}} \sum_{i=1}^{m-1} B_{i}^{2} \\
&+\frac{p}{2 \lambda} B_{m}^{2}+\left(\frac{h}{2 \lambda}-\frac{h}{2 \lambda^{\prime}}\right)\left(\sum_{i=1}^{m} Q_{i}-\lambda^{\prime} T\right)^{2} \\
&-\sqrt{\frac{2 K h p}{\lambda(h+p)}}\left(B_{m}+\sum_{i=1}^{m} Q_{i}\right)-T \sqrt{\frac{2 \lambda^{\prime} K h p}{h+p}} \\
&+\lambda^{\prime} T \sqrt{\frac{2 K h p}{\lambda(h+p)}} .
\end{aligned}
$$

Therefore, the problem (5) can be formulated as the following form:

$$
\begin{array}{rl}
\min _{m, Q_{i}, B_{i}} & F\left(Q_{1}, \ldots, Q_{m}, B_{1}, \ldots, B_{m}\right) \\
\text { s.t. } & Q_{1}+Q_{2}+\cdots+Q_{m-1}+B_{m-1}<\lambda^{\prime} T \\
& Q_{1}+Q_{2}+\cdots+Q_{m}>\lambda^{\prime} T \\
& Q_{i}, B_{i} \geq 0, \quad i=1, \ldots, m .
\end{array}
$$

For this problem, we have the following conclusion.
Theorem 3. For inventory model (54), suppose $m$ orders are made in the first stage. If $\mu_{2}<m<u_{1}+1$, then the optimal ordering sizes and the optimal maximum shortage level in the first stage are, respectively,

$$
\begin{aligned}
Q_{1} & =\frac{p \lambda^{\prime}(\alpha h T+\beta)}{h(h+p)\left[(m-1) \alpha+p \lambda^{\prime}\right]} \\
Q_{i} & =\frac{\lambda^{\prime}(\alpha h T+\beta)}{h\left[(m-1) \alpha+p \lambda^{\prime}\right]}, \quad i=2,3, \ldots, m \\
B_{i} & =\frac{\lambda^{\prime}(\alpha h T+\beta)}{(h+p)\left[(m-1) \alpha+p \lambda^{\prime}\right]}, \\
B_{m} & =\sqrt{\frac{2 \lambda K h}{p(h+p)}},
\end{aligned}
$$

where $\alpha=(h+p)\left(\lambda^{\prime}-\lambda\right), \beta=\sqrt{2 \lambda K h p(h+p)}, u_{1}=$ $\lambda^{\prime} T \sqrt{h p / 2 \lambda K(h+p)}, u_{2}=T \sqrt{\lambda h p / 2 K(h+p)}+h /(h+p)$.

Proof. For problem (54), since all the constraints are linear, if the problem has an optimal solution, then it is a KKT point which satisfies the following system:

$$
\begin{aligned}
& \begin{aligned}
& \frac{\partial F}{\partial Q_{1}} \\
&= \frac{h}{\lambda^{\prime}} Q_{1}+\left(\frac{h}{\lambda}-\frac{h}{\lambda^{\prime}}\right)\left(\sum_{i=1}^{m} Q_{i}-\lambda^{\prime} T\right) \\
&-\sqrt{\frac{2 K h p}{\lambda(h+p)}}=0, \\
& \frac{\partial F}{\partial Q_{i}} \\
&= \frac{h}{\lambda^{\prime}}\left(Q_{i}-B_{i-1}\right)+\left(\frac{h}{\lambda}-\frac{h}{\lambda^{\prime}}\right)\left(\sum_{i=1}^{m} Q_{i}-\lambda^{\prime} T\right) \\
&-\sqrt{\frac{2 K h p}{\lambda(h+p)}}=0, \quad i=2, \ldots, m, \\
& \frac{\partial F}{\partial B_{i}}=-\frac{h}{\lambda^{\prime}}\left(Q_{i+1}-B_{i}\right)+\frac{p}{\lambda^{\prime}} B_{i}=0, \\
& \frac{\partial F}{\partial B_{m}}=\frac{p}{\lambda} B_{m}-\sqrt{\frac{2 K h p}{\lambda(h+p)}}=0, \\
& Q_{1}+Q_{2}+\cdots+Q_{m-1}+B_{m-1}<\lambda^{\prime} T, i=1, \ldots, m-1, \\
& Q_{1}+Q_{2}+\cdots+Q_{m}>\lambda^{\prime} T, \\
& Q_{i}, B_{i} \geq 0, \quad i=1, \ldots, m,
\end{aligned}
\end{aligned}
$$


For the systems of equations, we can obtain that

$$
\begin{aligned}
Q_{1}= & \frac{p}{h+p} \\
& \cdot \frac{\lambda^{\prime}\left[h T(h+p)\left(\lambda^{\prime}-\lambda\right)+\sqrt{2 \lambda K h p(h+p)}\right]}{h\left[(m-1)(h+p)\left(\lambda^{\prime}-\lambda\right)+p \lambda^{\prime}\right]}, \\
Q_{i}= & \frac{\lambda^{\prime}\left[h T(h+p)\left(\lambda^{\prime}-\lambda\right)+\sqrt{2 \lambda K h p(h+p)}\right]}{h\left[(m-1)(h+p)\left(\lambda^{\prime}-\lambda\right)+p \lambda^{\prime}\right]}, i=2, \ldots, m, \\
B_{i}= & \frac{h}{h+p}, \\
B_{m}= & \sqrt{\frac{\lambda^{\prime}\left[h T(h+p)\left(\lambda^{\prime}-\lambda\right)+\sqrt{2 \lambda K h p(h+p)}\right.}{p(h+p)} .}, \\
& \frac{h\left[(m-1)(h+p)\left(\lambda^{\prime}-\lambda\right)+p \lambda^{\prime}\right]}{i=1, \ldots, m-1}
\end{aligned}
$$

Taking the inequality constraints into considerations, we have that

$$
\begin{gathered}
T \sqrt{\frac{\lambda h p}{2 K(h+p)}+\frac{h}{h+p}}<m \\
<\lambda^{\prime} T \sqrt{\frac{h p}{2 \lambda K(h+p)}}+1,
\end{gathered}
$$

i.e., $u_{2}<m<u_{1}+1$. The proof is completed.

By Theorem 3, under the Scenario $\sum_{i=1}^{m} Q_{i}>\lambda^{\prime} T$, we can obtain the following optimal ordering policy for problem (5).

$\operatorname{Policy}_{\mathrm{IV}}$

$$
Q=\frac{\lambda^{\prime}(\alpha h T+\beta)}{h\left[(m-1) \alpha+p \lambda^{\prime}\right]}
$$

$$
\begin{aligned}
Q_{1} & =\frac{p}{h+p} Q, \\
B & =\frac{h}{h+p} Q, \\
B_{m} & =\sqrt{\frac{2 \lambda K h}{p(h+p)}},
\end{aligned}
$$

which applies to the case that $u_{2}<m<u_{1}+1$.

The operating cost over the interval of length $T_{m}$ under this policy relative to the IBEOQ ordering policy is

$$
\begin{aligned}
F_{\mathrm{IV}}= & m K+\frac{p \lambda^{\prime}[m(h+p)-h](\alpha h T+\beta)^{2}}{2 h(h+p)^{2}\left[(m-1) \alpha+p \lambda^{\prime}\right]^{2}} \\
& +\frac{\lambda^{\prime}\left(\lambda^{\prime}-\lambda\right)[(h+p) \beta m-(h \beta+\gamma)]^{2}}{2 \lambda h(h+p)^{2}\left[(m-1) \alpha+p \lambda^{\prime}\right]^{2}} \\
& -\frac{\lambda^{\prime} \beta[m(h+p)-h](\alpha h T+\beta)}{\lambda h(h+p)^{2}\left[(m-1) \alpha+p \lambda^{\prime}\right]}-\frac{K h}{h+p} \\
& +\lambda^{\prime} T \sqrt{\frac{2 K h p}{\lambda(h+p)}}-T \sqrt{\frac{2 \lambda^{\prime} K h p}{h+p}},
\end{aligned}
$$

Now, we consider the optimal ordering times in the first stage under Policies $\pi_{\mathrm{IV}}$.

Similarly, for function $F_{\text {IV }}$ given by $(60)$, by

$$
F_{\text {IV }}^{\prime \prime}(m)=\frac{\alpha \lambda^{\prime} p(\beta+\alpha h T)^{2}}{h(h+p)\left(\lambda^{\prime} p+(m-1) \alpha\right)^{3}}>0,
$$

we know that $F_{\mathrm{IV}}(m)$ is a convex function in $m$, and its minimum can be obtained at the stationary point of the function; i.e.,

$$
\bar{m}_{\mathrm{IV}}=T \sqrt{\frac{\lambda^{\prime} h p}{2 K(h+p)}}+1-\frac{p\left(\lambda^{\prime}-\sqrt{\lambda \lambda^{\prime}}\right)}{\alpha}>u_{2} .
$$

Denote $m_{\mathrm{IV}_{1}}=\left\lfloor\bar{m}_{\mathrm{IV}}\right\rfloor, m_{\mathrm{IV}_{2}}=\left\lceil\bar{m}_{\mathrm{IV}}\right\rceil$. A similar discussion yields the optimal ordering times in the first stage

$$
m^{*}= \begin{cases}m_{\mathrm{IV}} & \text { if } u_{2}<m_{\mathrm{IV}_{1}} \leq m_{\mathrm{IV} 2}<u_{1}+1 \text { and } F_{\mathrm{IV}}\left(m_{\mathrm{IV}}\right) \leq F_{\mathrm{IV}}\left(m_{\mathrm{IV}_{2}}\right), \text { or } u_{2}<m_{\mathrm{IV}_{1}}<u_{1}+1 \leq m_{\mathrm{IV}_{2}}, \\ m_{\mathrm{IV}} & \text { if } m_{\mathrm{IV}_{1}} \leq u_{2}<m_{\mathrm{IV} 2}<u_{1}+1 \text { and } F_{\mathrm{IV}}\left(m_{\mathrm{IV}_{1}}\right)>F_{\mathrm{IV}}\left(m_{\mathrm{IV}_{2}}\right), \text { or } m_{\mathrm{IV}_{1}}<u_{2} \leq m_{\mathrm{IV}_{2}}<u_{1}+1\end{cases}
$$

Based on the discussion about three Scenarios above, we can present our algorithm for problem (5).

\subsection{Algorithm}

Step 1. Input value of parameters $K, \lambda, \lambda^{\prime}, h, p, T$.
Step 2. Compute $u_{1}=\lambda^{\prime} T \sqrt{h p / 2 \lambda K(h+p)}, u_{2}=$ $T \sqrt{\lambda h p / 2 K(h+p)}+h /(h+p), \alpha=(h+p)\left(\lambda^{\prime}-\lambda\right), \beta=$ $\sqrt{2 \lambda K h p(h+p)}, \overline{m_{\mathrm{I}}}=T \sqrt{\lambda^{\prime} h p / 2 K(h+p)}, \overline{m_{\mathrm{II}}}=h \lambda^{\prime} / \alpha+$ $\sqrt{\lambda^{\prime} h / 2 K p(h+p)}(p T-\beta / \alpha)$. 


$$
\begin{aligned}
& \overline{m_{\mathrm{III}}}=T \sqrt{\frac{\lambda^{\prime} h p}{2 K(h+p)}+\frac{h}{h+p},} \\
& \overline{m_{\mathrm{IV}}}=T \sqrt{\frac{\lambda^{\prime} h p}{2 K(h+p)}}+1-\frac{p\left(\lambda^{\prime}-\sqrt{\lambda \lambda^{\prime}}\right)}{\alpha} .
\end{aligned}
$$

Let

$$
\begin{aligned}
m_{\mathrm{I}_{1}} & =\left\lfloor\bar{m}_{\mathrm{I}}\right\rfloor, \\
m_{\mathrm{I}_{2}} & =\left\lceil\bar{m}_{\mathrm{I}}\right\rceil \\
m_{\mathrm{I}_{3}} & =\left\lfloor\min \left\{u_{1}, u_{2}\right\}\right\rfloor, \\
m_{\mathrm{II}_{1}} & =\left\lfloor\bar{m}_{\mathrm{II}}\right\rfloor, \\
m_{\mathrm{II}_{2}} & =\left\lceil\bar{m}_{\mathrm{II}}\right\rfloor, \\
m_{\mathrm{III}_{1}} & =\left\lfloor\bar{m}_{\mathrm{III}}\right\rfloor, \\
m_{\mathrm{III}_{2}} & =\left\lceil\bar{m}_{\mathrm{III}}\right\rceil, \\
m_{\mathrm{II}_{3}} & =\left\lceil u_{1}\right\rceil, \\
m_{\mathrm{II}_{4}} & =\left\lfloor u_{2}\right\rfloor, \\
m_{\mathrm{IV}_{1}} & =\left\lfloor\bar{m}_{\mathrm{IV}}\right\rfloor, \\
m_{\mathrm{IV}_{2}} & =\left\lceil\bar{m}_{\mathrm{IV}}\right\rceil .
\end{aligned}
$$

Compute $F_{\mathrm{I}}\left(m_{\mathrm{I}_{1}}\right), F_{\mathrm{I}}\left(m_{\mathrm{I}_{2}}\right), F_{\mathrm{II}}\left(m_{\mathrm{II}_{1}}\right), F_{\mathrm{II}}\left(m_{\mathrm{II}_{2}}\right), F_{\mathrm{II}}\left(m_{\mathrm{II}_{3}}\right)$, $F_{\mathrm{II}}\left(m_{\mathrm{II}_{4}}\right), F_{\mathrm{III}}\left(m_{\mathrm{III}_{1}}\right), F_{\mathrm{III}}\left(m_{\mathrm{III}_{2}}\right), F_{\mathrm{IV}}\left(m_{\mathrm{IV}_{1}}\right)$, and $F_{\mathrm{IV}}\left(m_{\mathrm{IV}_{2}}\right)$.

Step 3. If $F_{\mathrm{III}}\left(m_{\mathrm{III}_{1}}\right) \leq F_{\mathrm{III}}\left(m_{\mathrm{III}_{2}}\right)$, then

$$
\begin{aligned}
& m_{\mathrm{III}}^{*}=m_{\mathrm{III}_{1}}, \\
& F_{\mathrm{III}}^{*}=F_{\mathrm{III}}\left(m_{\mathrm{III}_{1}}\right),
\end{aligned}
$$

otherwise

$$
\begin{aligned}
& m_{\mathrm{III}}^{*}=m_{\mathrm{III}_{2}}, \\
& F_{\mathrm{III}}^{*}=F_{\mathrm{III}}\left(m_{\mathrm{III}_{2}}\right) .
\end{aligned}
$$

Step 4. If $m_{\mathrm{I}_{2}} \leq \min \left\{u_{1}, u_{2}\right\}$, and $F_{\mathrm{I}}\left(m_{\mathrm{I}_{2}}\right) \leq F_{\mathrm{I}}\left(m_{\mathrm{I}_{1}}\right)$, then

$$
\begin{aligned}
& m_{\mathrm{I}}^{*}=m_{\mathrm{I}_{2}}, \\
& F_{\mathrm{I}}^{*}=F_{\mathrm{I}}\left(m_{\mathrm{I}_{2}}\right),
\end{aligned}
$$

if $m_{\mathrm{I}_{1}}>\min \left\{u_{1}, u_{2}\right\}$

$$
\begin{aligned}
& m_{\mathrm{I}}^{*}=m_{\mathrm{I}_{3}}, \\
& F_{\mathrm{I}}^{*}=F_{\mathrm{I}}\left(m_{\mathrm{I}_{3}}\right),
\end{aligned}
$$

otherwise, $m_{\mathrm{I}}^{*}=m_{\mathrm{I}_{1}}, F_{\mathrm{I}}^{*}=F_{\mathrm{I}}\left(m_{\mathrm{I}_{1}}\right)$.
Step 5. (1) If $\beta<\alpha p T$,

(a) if $u_{2}<m_{\mathrm{II}_{1}} \leq m_{\mathrm{II}_{2}}<u_{1}$ and $F_{\mathrm{II}}\left(m_{\mathrm{II}_{1}}\right) \leq F_{\mathrm{II}}\left(m_{\mathrm{II}_{2}}\right)$, or $u_{2}<m_{\mathrm{II}_{1}}<u_{1} \leq m_{\mathrm{II}_{2}}$, then

$$
\begin{aligned}
& m_{\mathrm{II}}^{*}=m_{\mathrm{II}_{1}}, \\
& F_{\mathrm{II}}^{*}=F_{\mathrm{II}}\left(m_{\mathrm{II}_{1}}\right) ;
\end{aligned}
$$

(b) if $u_{2}<m_{\mathrm{II}_{1}} \leq m_{\mathrm{II}_{2}}<u_{1}$ and $F_{\mathrm{II}}\left(m_{\mathrm{II}_{1}}\right)>F_{\mathrm{II}}\left(m_{\mathrm{II}_{2}}\right)$, or $m_{\mathrm{II}_{1}} \leq u_{2}<m_{\mathrm{II}_{2}}<u_{1}$, then

$$
\begin{aligned}
& m_{\mathrm{II}}^{*}=m_{\mathrm{II}_{2}}, \\
& F_{\mathrm{II}}^{*}=F_{\mathrm{II}}\left(m_{\mathrm{II}_{2}}\right),
\end{aligned}
$$

otherwise, $F_{\mathrm{II}}^{*}=\infty$.

(2) If $\beta \geq \alpha p T$,

(a) if $m_{\mathrm{II}_{3}}<u_{2}$ and $m_{\mathrm{II}_{4}}>u_{1}$ and $F_{\mathrm{II}}\left(m_{\mathrm{II}_{3}}\right) \leq F_{\mathrm{II}}\left(m_{\mathrm{II}_{4}}\right)$, or $m_{\mathrm{II}_{4}} \leq u_{1}<m_{\mathrm{II}_{3}}<u_{2}$, then

$$
\begin{aligned}
& m_{\mathrm{II}}^{*}=m_{\mathrm{II}_{3}}, \\
& F_{\mathrm{II}}^{*}=F_{\mathrm{II}}\left(m_{\mathrm{II}_{3}}\right) ;
\end{aligned}
$$

(b) if $u_{2}>m_{\mathrm{II}_{3}}$, and $m_{\mathrm{II}_{4}}>u_{1}$ and $F_{\mathrm{II}}\left(m_{\mathrm{II}_{3}}\right)>F_{\mathrm{II}}\left(m_{\mathrm{II}_{4}}\right)$, or $u_{1} \leq m_{\mathrm{II}_{4}}<u_{2}<m_{\mathrm{II}_{3}}$, then

$$
\begin{aligned}
& m_{\mathrm{II}}^{*}=m_{\mathrm{II}_{4}}, \\
& F_{\mathrm{II}}^{*}=F_{\mathrm{II}}\left(m_{\mathrm{II}_{4}}\right),
\end{aligned}
$$

0therwise, $F_{\mathrm{II}}^{*}=\infty$.

Step 6. If $u_{2}<m_{\mathrm{IV}_{1}} \leq m_{\mathrm{II}_{2}}<u_{1}+1$ and $F_{\mathrm{IV}}\left(m_{\mathrm{IV}_{1}}\right) \leq$ $F_{\mathrm{IV}}\left(m_{\mathrm{IV}_{1}}\right)$, or $u_{2}<m_{\mathrm{IV}_{1}}<u_{1}+1 \leq m_{\mathrm{IV}_{2}}$, then

$$
\begin{aligned}
& m_{\mathrm{IV}}^{*}=m_{\mathrm{IV}_{1}}, \\
& F_{\mathrm{IV}}^{*}=F_{\mathrm{IV}}\left(m_{\mathrm{IV}_{1}}\right),
\end{aligned}
$$

If $u_{2}<m_{\mathrm{IV}_{1}} \leq m_{\mathrm{II}_{2}}<u_{1}+1$ and $F_{\mathrm{IV}}\left(m_{\mathrm{IV}_{1}}\right)>F_{\mathrm{IV}}\left(m_{\mathrm{IV}_{1}}\right)$, or $m_{\mathrm{IV}_{1}} \leq u_{2}<m_{\mathrm{IV}_{2}}<u_{1}+1$, then

$$
\begin{aligned}
& m_{\mathrm{IV}}^{*}=m_{\mathrm{IV}_{2}}, \\
& F_{\mathrm{IV}}^{*}=F_{\mathrm{IV}}\left(m_{\mathrm{IV}_{2}}\right),
\end{aligned}
$$

otherwise, $F_{\mathrm{IV}}^{*}=\infty$.

Step 7. Compare $F_{\mathrm{I}}^{*}, F_{\mathrm{II}}^{*}, F_{\mathrm{III}}^{*}$, and $F_{\mathrm{IV}}^{*}$

If $\min \left\{F_{\mathrm{I}}^{*}, F_{\mathrm{II}}^{*}, F_{\mathrm{III}}^{*}\right.$, and $\left.F_{\mathrm{IV}}^{*}\right\}=F_{\mathrm{I}}^{*}$, then, the optimal order times, the optimal order size from the second replenishment cycle to $\mathrm{m}$-th replenishment cycle, and the optimal maximum shortage level of $\mathrm{m}$-th replenishment cycle in the first stage are, respectively,

$$
\begin{aligned}
& m^{*}=m_{\mathrm{I}}^{*}, \\
& Q^{*}=\frac{\lambda^{\prime} T(h+p)}{m^{*}(h+p)-h}, \\
& B_{m}^{*}=\frac{h}{h+p} Q^{*},
\end{aligned}
$$

go to Step 8 . 
If $\min \left\{F_{\mathrm{I}}^{*}, F_{\mathrm{II}}^{*}, F_{\mathrm{III}}^{*}\right.$, and $\left.F_{\mathrm{IV}}^{*}\right\}=F_{\mathrm{II}}^{*}$, then, the optimal order times, the optimal order size from the second replenishment cycle to $\mathrm{m}$-th replenishment cycle, and the optimal maximum shortage levels of $\mathrm{m}$-th replenishment cycle in the first stage are, respectively,

$$
\begin{aligned}
m^{*} & =m_{\mathrm{III}}^{*}, \\
Q^{*} & =\frac{\lambda^{\prime}(\beta-\alpha p T)}{p\left(h \lambda^{\prime}-\alpha m^{*}\right)}, \\
B_{m}^{*} & =\sqrt{\frac{2 \lambda K h}{p(h+p)}},
\end{aligned}
$$

go to Step 8.

If $\min \left\{F_{\mathrm{I}}^{*}, F_{\mathrm{II}}^{*}, F_{\mathrm{III}}^{*}\right.$, and $\left.F_{\mathrm{IV}}^{*}\right\}=F_{\mathrm{III}}^{*}$, the optimal order times, the optimal order size from the second replenishment cycle to $\mathrm{m}$-th replenishment cycle, and the optimal maximum shortage level of $\mathrm{m}$-th replenishment cycle in the first stage are, respectively,

$$
\begin{aligned}
& m^{*}=m_{\mathrm{I}}^{*}, \\
& Q^{*}=\frac{\lambda^{\prime} T(h+p)}{m^{*}(h+p)-h}, \\
& B_{m}^{*}=\sqrt{\frac{2 \lambda K h}{p(h+p)}},
\end{aligned}
$$

go to Step 8.

If $\min \left\{F_{\mathrm{I}}^{*}, F_{\mathrm{II}}^{*}, F_{\mathrm{II}}^{*}\right.$, and $\left.F_{\mathrm{IV}}^{*}\right\}=F_{\mathrm{IV}}^{*}$, the optimal order times, the optimal order size from the second replenishment cycle to $\mathrm{m}$-th replenishment cycle, and the optimal maximum shortage level of $\mathrm{m}$-th replenishment cycle in the first stage are, respectively,

$$
\begin{aligned}
& m^{*}=m_{\mathrm{IV}}^{*}, \\
& Q^{*}=\frac{\lambda^{\prime}(\beta+\alpha h T)}{h\left[p \lambda^{\prime}+\alpha\left(m^{*}-1\right)\right]}, \\
& B_{m}^{*}=\sqrt{\frac{2 \lambda K h}{p(h+p)}},
\end{aligned}
$$

go to Step 8 .

Step 8. The optimal order size of the first replenishment cycle and the optimal maximum shortage level of the first replenishment cycle (m-1)-th replenishment cycle in the first stage are, respectively,

$$
\begin{aligned}
Q_{1}^{*} & =\frac{p}{h+p} Q^{*}, \\
B^{*} & =\frac{h}{h+p} Q^{*},
\end{aligned}
$$

and terminate.

\section{Computational Experiments and Sensitivity Analysis}

In this section, we will perform some numerical experiments to test the validity of model (5) and the efficiency of the proposed algorithm.

Example 1. Consider the inventory system with the following parameters: $\lambda^{\prime}=15, \lambda=10, K=10, T=4, h=3, p=1$.

For this inventory system, direct computing gives that

$$
\begin{aligned}
\alpha & =20, \\
\beta & =48.9898, \\
u_{1} & =3.67, \\
u_{2} & =3.20, \\
\overline{m_{\mathrm{I}}} & =3.75, \\
\overline{m_{\mathrm{I}}} & =3, \\
\overline{m_{\mathrm{II}}} & =3.41, \\
\overline{m_{\mathrm{IV}}} & =3.86,
\end{aligned}
$$

Obviously, $\beta<\alpha p T$, and $\left\lfloor\overline{m_{\mathrm{II}}}\right\rfloor<u_{2},\left\lceil\overline{m_{\mathrm{II}}}\right\rceil>u_{1}$, by Algorithm, Policy $\pi_{\text {II }}$ does not apply and the operating cost under the other three policies relative to the IEOQ ordering policy are, respectively, $\{0.1923,0,-0.202\}$, so the optimal policy is Policy $\pi_{\mathrm{IV} 2}$ and the inventory cost saving over the IEOQ ordering policy by the Policy $\pi_{\text {IV }}$ is 0.202 ; the optimal ordering times, the ordering size, and the optimal maximum shortage level in the first stage are, respectively,

$$
\begin{aligned}
& m^{*}=4, \\
& Q^{*}=19.2660, \\
& Q_{1}^{*}=4.8165, \\
& B^{*}=14.4495, \\
& B_{m}^{*}=12.2474 .
\end{aligned}
$$

To show the length $T$ of the first stage on ordering policy, we reconsider the variation of the inventory cost saving under the optimal ordering policies when $T$ varies from 0 to 30 on the problem. The numerical results are shown in Figure 5, from which we can see that the inventory cost saving under the optimal ordering policy over the IEOQ ordering policy increases cyclically to a constant and is nonnegative, which means that the ordering policy given by Algorithm is superior to the IEOQ ordering policy. Further, the numerical experiments show that the ordering Policies $\pi_{\mathrm{I}}, \pi_{\mathrm{II}}$, and $\pi_{\text {III }}$ almost never apply for the problem and therefore the remaining stock at $T$ is always positive for $T \in(0,30]$. See Figure 6.

Example 2. Consider the inventory system in which $\lambda^{\prime}=$ $15, K=10, T=15, h=3, p=1$. 


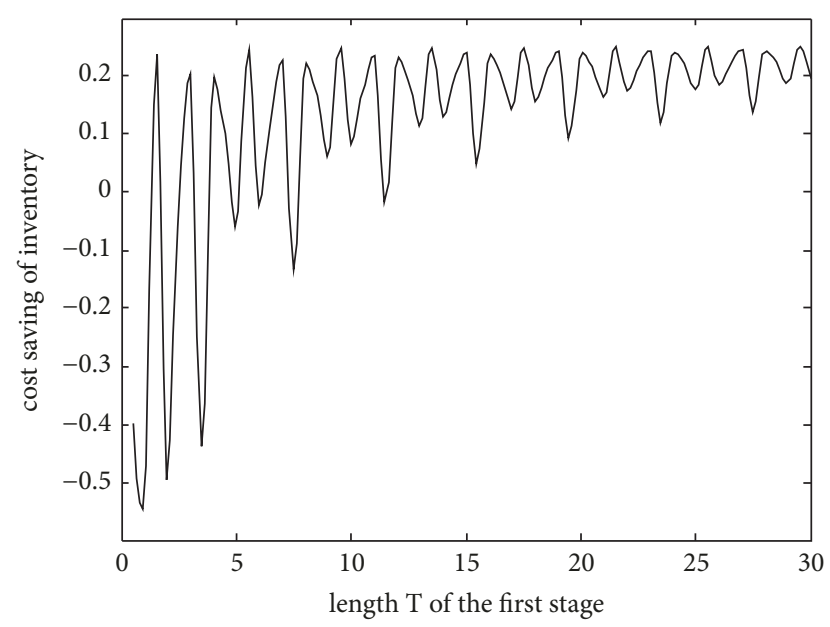

FIgURE 5: Variation of cost saving w.r.t. $T$ in Example 1.

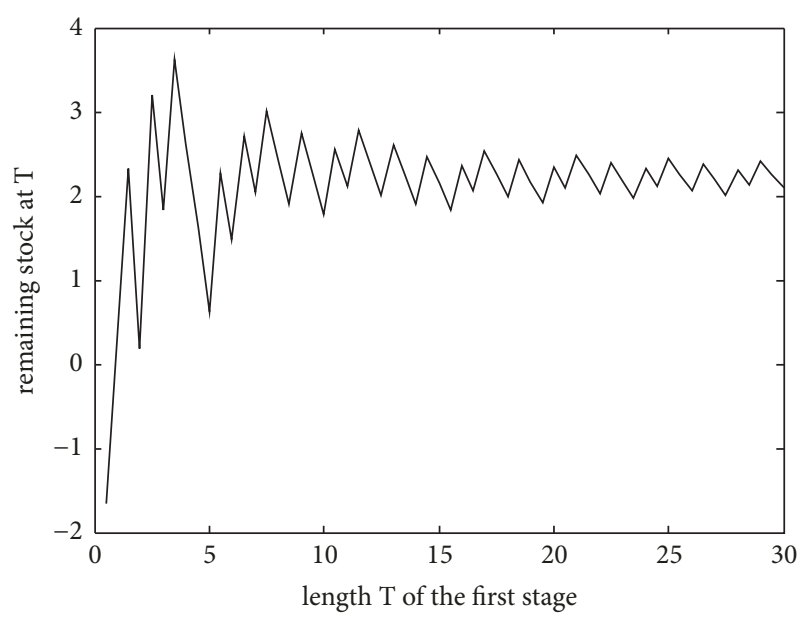

FIgURE 6: Variation of remaining stock at $T$ in Example 1.

For this inventory system, we test the effect of ratio $\lambda^{\prime} / \lambda$ on the inventory cost saving using Algorithm. To this end, we allow $\lambda^{\prime}$ to vary from $\lambda$ to $3 \lambda$. The numerical results are shown in Figure 7, from which we can see that the inventory cost saving is monotonically increasing with the ratio $\lambda^{\prime} / \lambda$. Combining with the numerical results for Example 1 that the remaining stock at $T$ under the optimal ordering policies is always positive, as shown in Figure 6, we can see that the remaining stock at $T$ is cyclical w.r.t. the length $T$ of the first stage and the ratio $\lambda^{\prime} / \lambda$.

For the inventory model (5) with $\lambda^{\prime}>\lambda$, the numerical results show that almost all optimal ordering policies are Policy $\pi_{\mathrm{IV}}$, whereas Policy $\pi_{\mathrm{II}}$ never applies. Further, the optimal ordering policy given by Algorithm is superior to the IEOQ ordering policy.

\section{Conclusion and Extensions}

This study investigated an optimization model for the inventory mechanism with step-shaped demand and backordering. On the basis of the inventory cost analysis under different

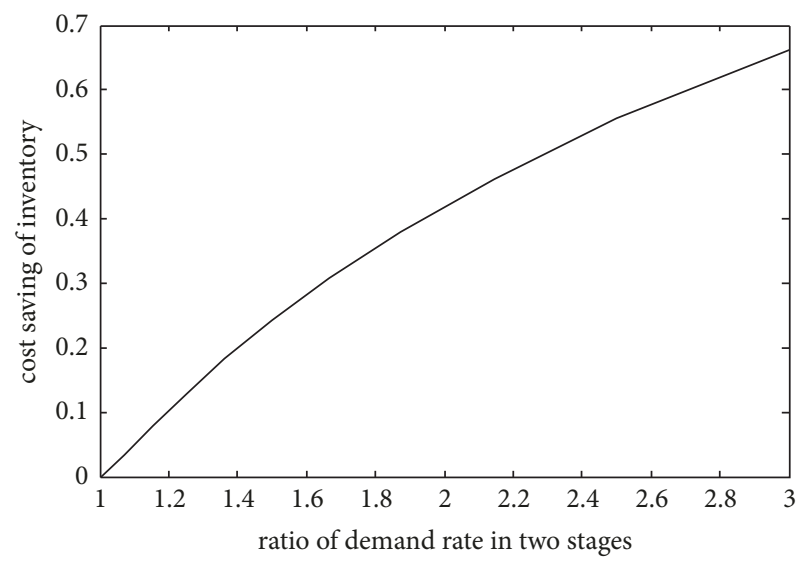

Figure 7: Variation of cost saving w.r.t. ratio $\lambda^{\prime} / \lambda$ in Example 2.

ordering policies, we presented a closed-form solution to the model. From the obtained results, we demonstrate that the economic order quantity is not necessarily an optimal ordering policy for the concerned inventory model. For the model, there are four possible optimal ordering policies, namely, Policies $\pi_{\mathrm{I}}, \pi_{\mathrm{II}}, \pi_{\mathrm{III}}$, and $\pi_{\mathrm{IV}}$. Our numerical results show that Policy $\pi_{\mathrm{II}}$ never applies. If this can be proved or confirmed in theory, then the given solution method can be simplified.

\section{Data Availability}

The data used in our numerical experiments are taken randomly, and all used data released in this paper can be used directly.

\section{Conflicts of Interest}

The authors declare that they have no conflicts of interest.

\section{Acknowledgments}

This work is supported by the Natural Science Foundation of China (11671228).

\section{References}

[1] F. W. Harris, "How many parts to make at once," Factory, the magazine of management, vol. 10, no. 2, pp. 135-136, 1913.

[2] R. H. Wilson, "A scientific routine for stock control," Harvard Business Review, vol. 13, pp. 116-128, 1934.

[3] M. Bakker, J. Riezebos, and R. H. Teunter, "Review of inventory systems with deterioration since 2001," European Journal of Operational Research, vol. 221, no. 2, pp. 275-284, 2012.

[4] S. Nahmias, "Production and operations analysis," Journal of Operational Research Society, 2008, McGraw-Hill College.

[5] C. D. J. Waters, Inventory Control and Management, Wiley, New Jersey, NJ, USA, 2003.

[6] M. Khouja and S. Park, "Optimal lot sizing under continuous price decrease,” Omega , vol. 31, no. 6, pp. 539-545, 2003. 
[7] Y. Wang, H. Gao, and W. Xing, "Optimal replenishment and stocking strategies for inventory mechanism with a dynamically stochastic short-term price discount," Journal of Global Optimization, vol. 70, no. 1, pp. 27-53, 2018.

[8] H. M. Wagner and T. M. Whitin, "Dynamic version of the economic lot size model," Management Science, vol. 5, no. 1, pp. 89-96, 1958.

[9] M. Resh, M. Friedman, and L. C. Barbosa, "On a general solution of the deterministic lot size problem with timeproportional demand," Operations Research, vol. 24, no. 4, pp. 718-725, 1976.

[10] W. A. Donaldson, "Inventory replenishment policy for a linear trend in demand - an analytical solution," Operational Research Quarterly (1970-1977), vol. 28, no. 3, p. 663, 1977.

[11] U. Dave, "A deterministic lot size inventory model with shortages and a linear trend in demand," Naval Research Logistics (NRL), vol. 36, no. 4, pp. 507-514, 1989.

[12] U. Dave, "On a heuristic inventory-replenishment rule for items with a linearly increasing demand incorporating shortages," Journal of the Operational Research Society, vol. 40, no. 9, pp. 827-830, 1989.

[13] J. K. Dey, S. Kar, and M. Maiti, "An interactive method for inventory control with fuzzy lead-time and dynamic demand," European Journal of Operational Research, vol. 167, no. 2, pp. 381-397, 2005.

[14] C. Muriana, "An EOQ model for perishable products with fixed shelf life under stochastic demand conditions," European Journal of Operational Research, vol. 255, no. 2, pp. 388-396, 2016.

[15] J.-W. Wu, C. Lin, B. Tan, and W.-C. Lee, "An EOQ inventory model with time-varying demand and Weibull deterioration with shortages," International Journal of Systems Science, vol. 31, no. 6, pp. 677-683, 2000.

[16] X. Yan, "An EOQ model for perishable items with freshnessdependent demand and partial backlogging," International Journal of Control and Automation, vol. 5, no. 4, pp. 19-38, 2012.

[17] R. C. Baker and T. L. Urban, "A deterministic inventory system with an inventory-level-dependent demand rate," Journal of the Operational Research Society, vol. 39, no. 9, pp. 883-884, 1988.

[18] T. K. Datta and A. K. Pal, "A note on an inventory model with inventory-level-dependent demand rate," Journal of the Operational Research Society, vol. 41, no. 10, pp. 971-975, 1990.

[19] T. L. Urban, "An inventory model with an inventory-leveldependent demand rate and relaxed terminal conditions," Journal of the Operational Research Society, vol. 43, no. 7, pp. 721-724, 1992.

[20] B. N. Mandal and S. Phaujdar, "An inventory model for deteriorating items and stock-dependent consumption rate," Journal of the Operational Research Society, vol. 40, pp. 483-488, 1989.

[21] Y. Wang, L. Caccetta, and G. Zhou, "Convergence analysis of a block improvement method for polynomial optimization over unit spheres," Numerical Linear Algebra with Applications, vol. 22, no. 6, pp. 1059-1076, 2015.

[22] Y. Wang, X. Sun, and F. Meng, "On the conditional and partial trade credit policy with capital constraints: A Stackelberg Model," Applied Mathematical Modelling, vol. 40, no. 1, pp. 1-18, 2016. 


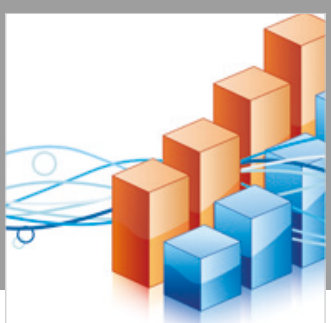

Advances in

Operations Research

\section{-n-m}
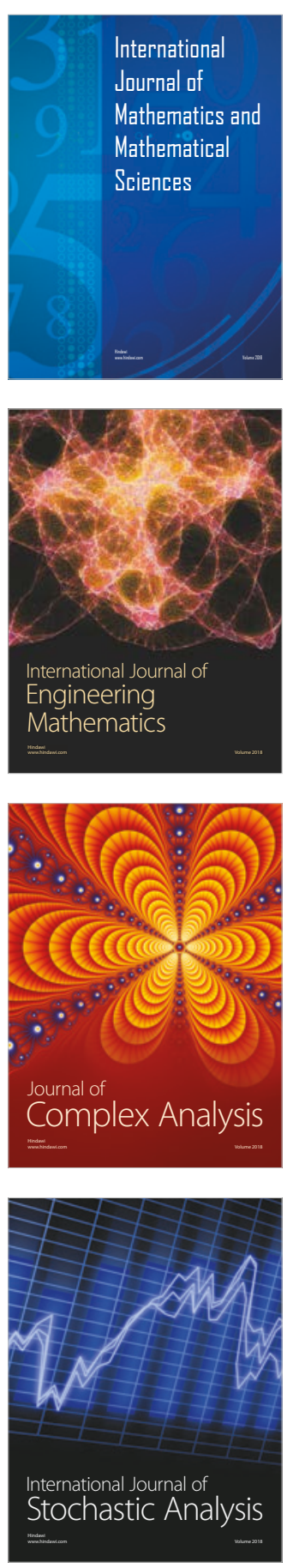
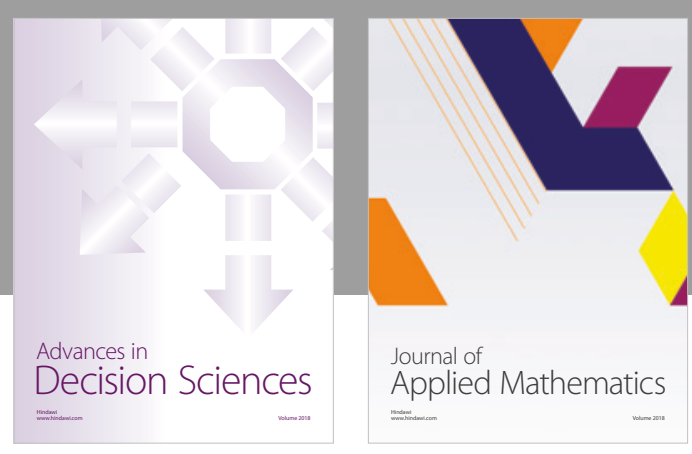

Journal of

Applied Mathematics
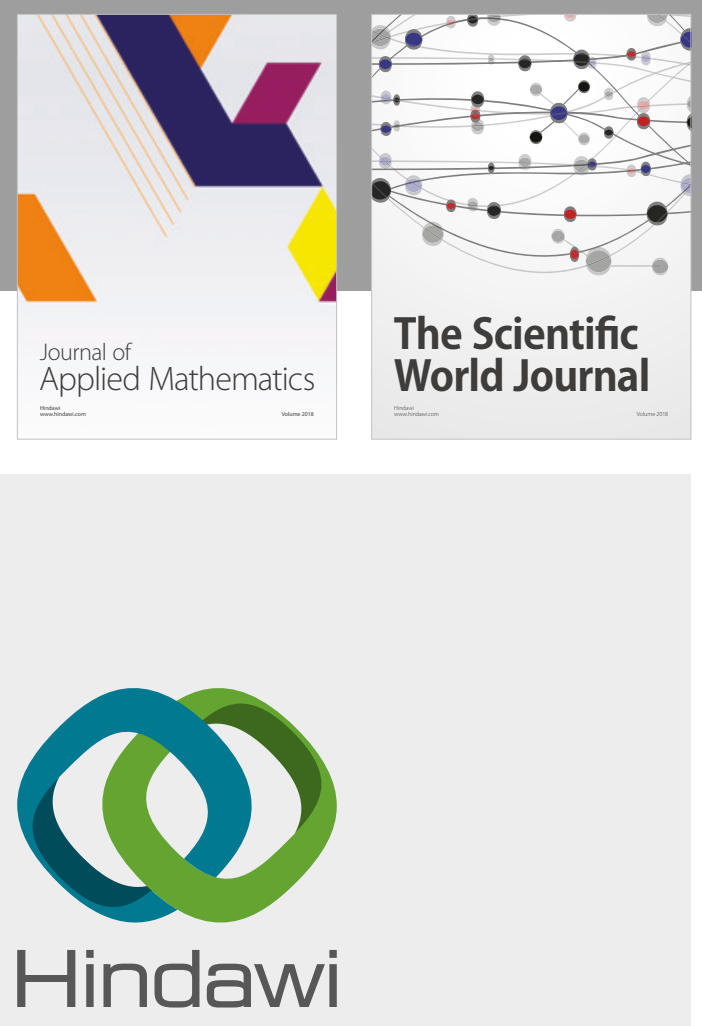

Submit your manuscripts at

www.hindawi.com

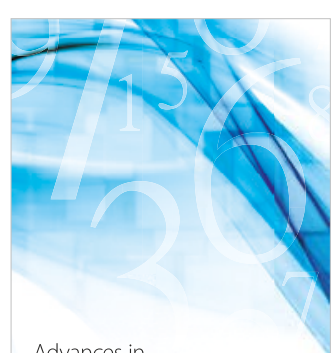

Advances in
Numerical Analysis
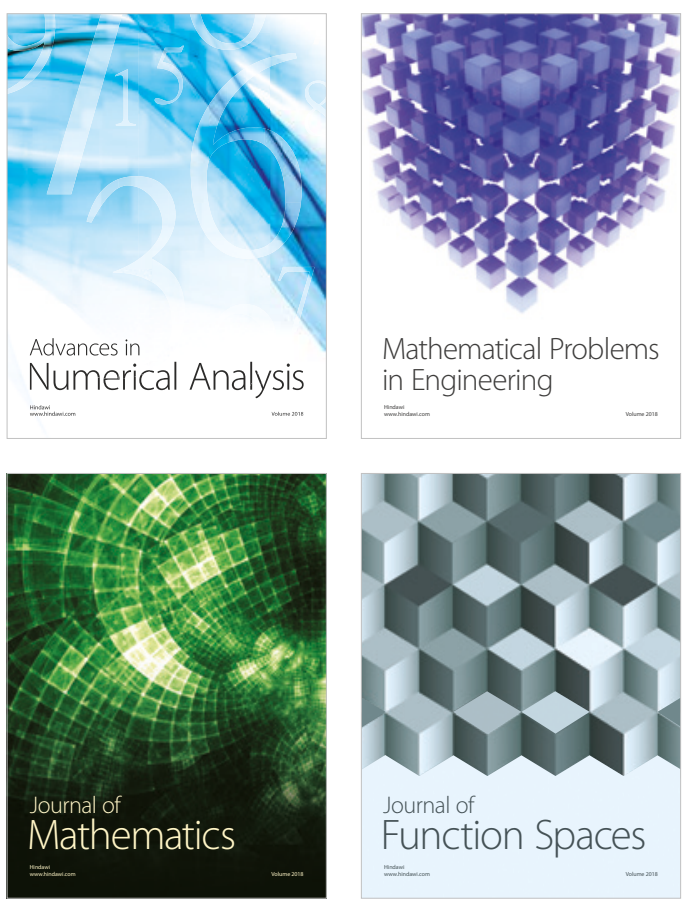

Mathematical Problems in Engineering

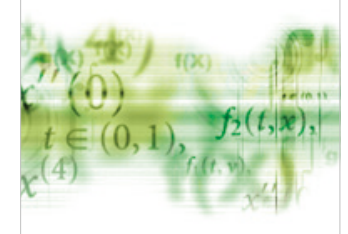

International Journal of

Differential Equations

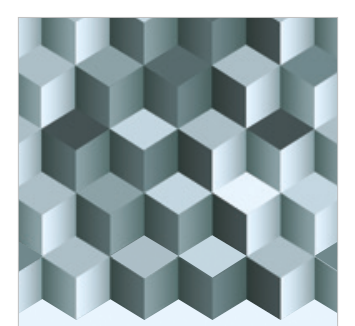

Journal of

Function Spaces

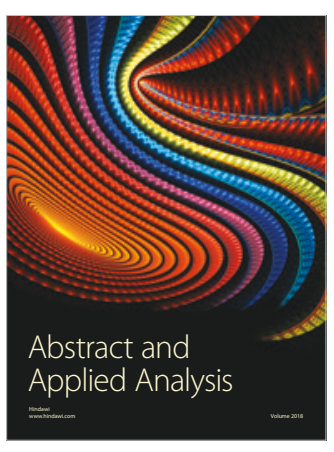

The Scientific

World Journal

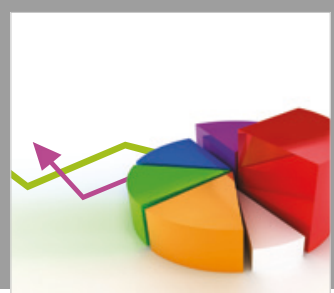

Journal of

Probability and Statistics
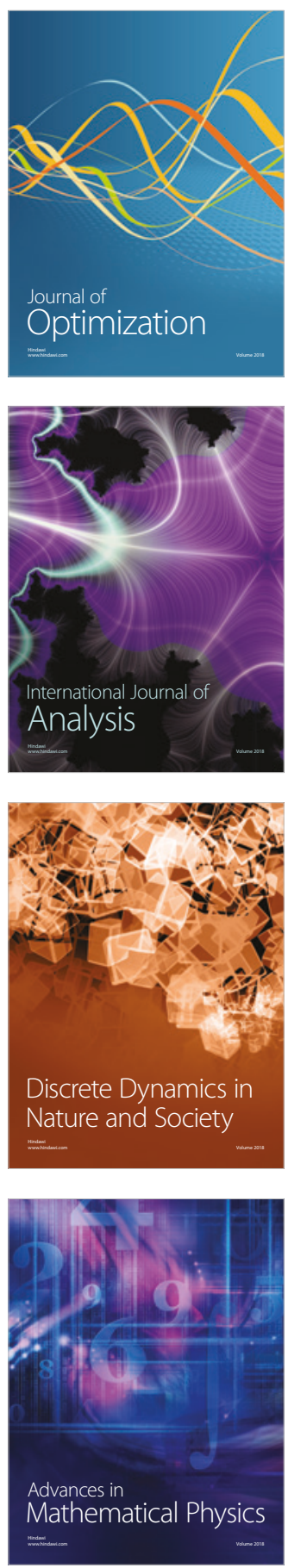